\title{
Cyclic Hydroxamic Acid Content and its Temporal Changes in Five Commercial Maize (Zea Mays) Hybrids
}

\author{
P. Makleit ${ }^{1 *}$, A. NAGY ${ }^{2}$, S. Veres ${ }^{1}$ and A. FónagY ${ }^{3}$ \\ ${ }^{1}$ University of Debrecen, Faculty of Agricultural and Food Sciences and Environmental Management, \\ Institute of Crop Sciences, H-4032 Debrecen, Böszörményi út 138. Hungary \\ ${ }^{2}$ University of Debrecen, Faculty of Agricultural and Food Sciences and Environmental Management, \\ Institute of Plant Protection, H-4032 Debrecen, Böszörményi út 138. Hungary \\ ${ }^{3}$ Plant Protection Institute, Centre for Agricultural Research, Hungarian Academy of Sciences, \\ H-1022 Budapest, Herman Ottó u. 15.
}

(Received 22 November 2017; Accepted 13 April 2018;

Communicated by S. Gottwald)

The concentration (in $\mathrm{mg} \mathrm{kg}^{-1}$ fresh weight) of two main hydroxamates, 2,4-dihydroxy7-methoxy-1,4-benzoxazin-3-one (DIMBOA) and 2,4-dihydroxy-1,4-benzoxazin-3-one (DIBOA), and their temporal changes were simultaneously investigated using HPLC analysis in the leaves and roots of five Pioneer ${ }^{\circledR}$ maize (Zea mays L.) hybrids to select hybrids with higher hydroxamate contents. Although significant differences were found among hybrids in leaves, youngest leaves and roots, none of them showed unambiguously higher hydroxamate contents. However, the age of the organs and the plants significantly affected hydroxamate content. DIMBOA content of leaves decreased with increasing organ and plant age. DIBOA content varied among the hybrids, but generally decreased in the initial phase and then increased. In the roots, DIMBOA content decreased during the 21-day study and although DIBOA content did not show a clear temporal tendency, differences among hybrids were detected. According to current results, hydroxamate content temporally decreases in hybridspecific patterns, which should be considered when establishing a proper sampling time frame.

Keywords: DIMBOA, DIBOA, effect of plant age, effect of organ age, Pioneer ${ }^{\circledR}$ maize hybrids

\section{Introduction}

Host plant resistance to insect pests consists of numerous chemical and biochemical factors with a capacity to limit insect damage. Plants produce thousands of unique low-mass natural products, referred to as secondary metabolites. These compounds are generally non-essential for the basic metabolic processes of plants, but may substantially improve their defence capacity against microbial attack, herbivore predation and control allelopathic interactions (McMullen et al. 2009). These factors are stored in sequestered forms and transformed into active structures upon insect infestation and/or in cases of tissue

\footnotetext{
*Corresponding author; E-mail: pmakleit@agr.unideb.hu
} 
damage. Besides secondary metabolites, various enzymatic mechanisms improve the plant resistance to pests (superoxide dismutases, NADPH oxidases, glutathione transferases) (Sytykiewicz 2014, 2016; Sytykiewicz et al. 2014).

Cyclic hydroxamic acids (hydroxamates) are plant born benzoxazinoids. These secondary metabolites are produced predominantly by the species of the Poaceae family (Niemeyer 2009). The hydroxamate content is species and hybrid specific (Zuniga et al. 1991) and also depends on various other parameters, e.g. plant and organ age, environmental conditions and stress factors. The role of these factors has not been fully revealed until now because the studies generally focused on the amount and changes of hydroxamate content of a given organ (roots or leaves) or the whole shoot of a given hybrid (Long et al. 1974; Reid et al. 1991; Xie et al. 1991 a, b; Xie et al. 1992; Assabgui et al. 1993; Assabgui et al. 1995 a, b; Abel et al. 2000).

Hydroxamates are the key defence chemicals of cereals: they protect the plants against pathogens and pests, especially in the early stages of development (Niemeyer 2009). The main hydroxamate in maize (Zea mays L.) and wheat is 2- $\beta$-D-glucopyranosyloxy-4hydroxy-7-methoxy-1,4-benzoxazine-3-one (DIMBOA-Glc), while 2- $\beta$-Dglucopyranosyloxy-4-hydroxy-1,4-benzoxazine-3-one (DIBOA-Glc) is present in lower amounts. In injured plants, the glycosides are hydrolyzed by $\beta$-glycosidase, yielding the respective aglycones. Primarily the 2,4-dihydroxy-7-methoxy-1,4-benzoxazin-3-one (DIMBOA) plays different roles in the Poaceae family by exerting both toxic and antifeedant effects on pests, as summarized by Niemeyer (2009). The rising costs of pesticides, their undesirable effects on the environment and the increasing number of strains of insect species resistant to them have led to renewed efforts to identify and better exploit host plant resistance to pests and diseases. It is essential to understand and develop knowledge of factors determining the hydroxamate content and hydroxamate synthesis potentials of plants of different varieties/hybrids to allow for meaningful use of their natural capacities.

A case study presented here on five Pioneer ${ }^{\circledR}$ maize hybrids, in which the amount and the change of the cyclic hydroxamates were investigated simultaneously for different organs, in order to select the hybrid with the best resistance capacity.

\section{Materials and Methods}

\section{Plant material and sampling}

Test material and relevant documentation were provided by Pioneer Hi-Bred Zrt. (Hungary). Studies were performed on various FAO number Pioneer ${ }^{\circledR}$ maize hybrids: PR38A79 (FAO 310), P9578 (FAO320), P9494 (FAO390), PR36K67 (FAO 480) and PR36V52 (FAO 430) hybrids. Maize seedlings were grown in rearing chambers in nutrient solution, under $300 \mu \mathrm{mol} \mathrm{m} \mathrm{m}^{-2} \mathrm{~s}^{-1}$ light intensity, 25/20 ${ }^{\circ} \mathrm{C}$ (Light/Dark) temperature, $16 \mathrm{~h} / 8 \mathrm{~h}$ (Light/Dark) periodicity and $65-75 \%$ relative humidity. The nutrient solution was made according to Treeby et al. (1989) with addition of iron in the form of Fe(III)-EDTA in quantity of $10^{-4}$ mole litre ${ }^{-1}$. The medium was changed every three days and roots were aerated. This allowed sampling of the youngest and finest root tips, since that is not pos- 
sible if grown in soil. During nutrient solution cultivation, the number of seedlings was 10 per pot for each hybrid, in separate pots, in order to supply fresh samples suitable for Reverse Phase-High Performance Liquid Chromatography (RP-HPLC) measurements for quantification. From each hybrid, 4 replicates were prepared to ensure a sufficient amount of sampling material for the four sampling times. Samples were taken from young plants on the $3^{\text {rd }}, 9^{\text {th }}, 15^{\text {th }}$ and $21^{\text {st }}$ days after emerging, from the youngest parts of the roots and from three different leaves from the shoots. In the case of the roots, samples were taken from primary roots. The length of root segments was about 2 centimetres. Samples were taken from a single plant once, because the resulting damage can induce higher rates of hydroxamates (Morse et al. 1991). Sampling was performed randomly from the pots and 6 individuals were selected from each cultivar and at a time. These individuals originated from 3 separate pots. On the $3^{\text {rd }}$ day, the $1^{\text {st }}$ and $2^{\text {nd }}$; on the $9^{\text {th }}$ day, the $1^{\text {st }}, 2^{\text {nd }}$, and $3^{\text {rd }}$; on the $15^{\text {th }}$ day, the $1^{\text {st }}, 3^{\text {rd }}$ and $5^{\text {th }}$; and on the $21^{\text {st }}$ day, the $1^{\text {st }}, 4^{\text {th }}$ and $7^{\text {th }}$ leaves were sampled. The sampling was standardized by cutting tips (about 2 centimetres long part of the leaves' apices) because this part can be easily sampled, independent of the size and the age of the organ. Samplings were done very carefully, but quickly, to avoid any degradation of target compounds. Each sample was weighed, then placed into labelled sachets and sealed. The samples were immediately placed into $-80{ }^{\circ} \mathrm{C}$ and stored. In the cases of the PR38A79 and P9494 hybrids, on the $3^{\text {rd }}$ day, the 3rd leaves were not fully developed yet and on the $21^{\text {st }}$ day, the youngest leaves were uniformly the $6^{\text {th }}$ leaves, because the $7^{\text {th }}$ did not reach the required development for sampling (parallels). Since roots were also sampled, altogether $2 \times 360$ samples were taken for RP-HPLC measurements. Experiments were conducted in 2014-2015 in the rearing chambers of the University of Debrecen, Faculty of Agricultural and Food Sciences and Environmental Management, Institute of Crop Sciences, Department of Agricultural Botany, Crop Physiology and Biotechnology. HPLC measurements were carried out at the University of Debrecen, Faculty of Science and Technology, Institute of Chemistry, Department of Organic Chemistry.

\section{Sample preparation}

The main goal was the qualification and quantification of 2,4-dihydroxy-7-methoxy-1,4benzoxazin-3-one (DIMBOA) and 2,4-dihydroxy-1,4-benzoxazin-3-one (DIBOA) content by using RP-HPLC-analysis. For sample preparation, the method of Lyons et al. (1988) was applied, which is based on the decomposition of glucosides into their aglucones.

\section{Purification and isolation of standards}

The procedure to obtain DIMBOA and DIBOA standards was performed according to Hartenstein et al. (1992). The first was done from etiolated maize plants, while the latter was done from etiolated rye. DIMBOA and DIBOA were identified by melting point measurements and by ${ }^{1} \mathrm{H}$ NMR spectra recorded for $\mathrm{CD}_{3} \mathrm{OD}$ solutions with a Bruker 360 (360 $\mathrm{MHz})$ spectrometer. Chemical shifts were referenced to $\mathrm{CH}_{3} \mathrm{OH}(\delta 3.31 \mathrm{ppm})$. The established melting point was $168.0-169.0^{\circ} \mathrm{C}$ for DIMBOA and $163.5-165.0^{\circ} \mathrm{C}$ for DIBOA. 


\section{RP-HPLC-analysis}

A Thermo Accela (fully automatic) unit equipped with a reverse phase C18 column (Lichrospher, $50 \times 2.1 \mathrm{~mm}$ ) was used. The volume of the respective samples was $20 \mu \mathrm{l}$. The variable wavelength detector was set to $280 \mathrm{~nm}$. Solvents of the mobile phase were: A: $1 \%$ acetic acid, B: $100 \%$ methanol. The flow rate was $1.0 \mathrm{ml} \mathrm{min}{ }^{-1}$. The ratio of the mobile phase (A:B) was 9:1 at the beginning, which reached a 1:1 ratio at the $3^{\text {rd }}$ minute using a linear gradient and the runs were carried out with this ratio over the next 8 minutes. Finally, the ratio of the solvents reached their initial value in the last 30 seconds. The samples were injected every 20 minutes. At the launch of every set of measurements, solutions of pure DIMBOA and DIBOA were used to create standard curves. The standard solutions were made from crystalline material stored at $-20{ }^{\circ} \mathrm{C}$ to avoid sample degradation.

\section{Data analysis}

The mean hydroxamate (DIMBOA and DIBOA) contents of shoots and roots of five studied hybrids were compared with one-way ANOVA. Bonferroni post-hoc test was applied for comparison among means if significant differences were found. The comparison was performed both on all samples of different organs and only on the youngest leaves, sampled at their first appearance. The Kolmogorov-Smirnov test for normality and the Levene-test for homogeneity of variances was used to check that the assumptions of the ANOVA were met. Based on the results of these tests, the data from the roots passed the tests, but $\lg (1+\mathrm{xi})$ transformation was applied in the case of shoots to achieve variance homogeneity. The effect of the organ and plant ages and the hybrids on the hydroxamate content were analysed by repeated measures ANOVA, where the age (day) was the within-, and the hybrid was the between-type effect (Reiczigel et al. 2007). All statistical analysis was performed using SPSS 21.0 statistical software (Ketskeméty et al. 2011).

\section{Results}

\section{Comparison of hybrids cyclic hydroxamic acid content}

The DIMBOA content of shoots was an order of magnitude higher than the DIBOA content in all studied hybrids. The DIMBOA content of shoots differed significantly for all five hybrids: hybrid P9494 was significantly (Bonferroni post-hoc test $\mathrm{p}<0.01$ ) higher than that of the P38A79, P9578 and PR36K67 hybrids. DIMBOA content of the shoots of the PR36V52 hybrid showed intermediate values. The DIBOA content of shoots also differed significantly among hybrids, where the hybrid P9494 was the highest again, while almost all of the other hybrids' shoots had significantly lower DIBOA contents (Table 1).

Similar to that of the shoots, the DIMBOA content of the roots was much higher than the DIBOA content. DIMBOA content of the roots of the PR36K67 hybrid was remark- 
Table 1. The mean hydroxamates $(\mathrm{cHx})$ content $( \pm \mathrm{SE} / \mathrm{SD})$ of hybrids on the basis of all measured shoot samples (column A), root samples (column B) and on the basis of the youngest parts (at first appearance) of the shoots (column C)

\begin{tabular}{|c|c|c|c|c|}
\hline \multirow{2}{*}{ Hybrids } & A & B & C \\
\cline { 3 - 5 } & \multicolumn{3}{|c|}{ DIMBOA } \\
\hline H1 & PR38A79 & $257.17 \pm 399.08 \mathrm{a}$ & $448.55 \pm 231.73 \mathrm{a}$ & $309.35 \pm 460.05 \mathrm{a}$ \\
\hline H2 & P9578 & $204.53 \pm 366.33 \mathrm{a}$ & $403.42 \pm 146.74 \mathrm{a}$ & $336.92 \pm 494.30 \mathrm{ab}$ \\
\hline H3 & P9494 & $454.66 \pm 504.00 \mathrm{~b}$ & $290.94 \pm 242.61 \mathrm{a}$ & $448.05 \pm 501.79 \mathrm{ab}$ \\
\hline H4 & PR36K67 & $257.62 \pm 452.43 \mathrm{a}$ & $662.65 \pm 240.19 \mathrm{~b}$ & $495.60 \pm 646.58 \mathrm{ab}$ \\
\hline H5 & PR36V52 & $292.21 \pm 438.58 \mathrm{ab}$ & $293.38 \pm 145.61 \mathrm{a}$ & $517.78 \pm 610.99 \mathrm{~b}$ \\
\hline \multicolumn{5}{|c|}{} \\
\hline H1 & PR38A79 & $35.28 \pm 65.17 \mathrm{ac}$ & DIBOA \\
\hline H2 & P9578 & $51.94 \pm 49.52 \mathrm{ab}$ & $1.6 \pm 3.22 \mathrm{a}$ & $39.02 \pm 39.81 \mathrm{a}$ \\
\hline H3 & P9494 & $78.18 \pm 93.39 \mathrm{~b}$ & $6.31 \pm 4.48 \mathrm{~b}$ & $59.28 \pm 83.89 \mathrm{a}$ \\
\hline H4 & PR36K67 & $26.64 \pm 38.07 \mathrm{ac}$ & $1.62 \pm 2.00 \mathrm{a}$ & $28.44 \pm 45.29 \mathrm{a}$ \\
\hline H5 & PR36V52 & $31.88 \pm 54.09 \mathrm{c}$ & $3.1 \pm 2.92 \mathrm{ab}$ & $49.31 \pm 77.64 \mathrm{a}$ \\
\hline
\end{tabular}

ably high, while the other hybrids did not show significant differences. However, significant differences were detected in the DIBOA content of the hybrids' roots. (Table 1)

First and last in the shoots, the P9494 hybrid represented the highest value, while in case of roots, the PR36K67 hybrid showed the highest DIMBOA content. Considering DIBOA content, hybrid P9494 was the highest in both shoots and roots.

The hydroxamate content of the youngest leaves, which were sampled at their first appearance, was also examined. In the youngest leaves, the DIBOA content - as in the roots and in the whole shoots - was much lower than the DIMBOA content (Table 1). The mean DIMBOA content of young leaves varied in the same range as that of all leaves, but the deviation of the values was higher. Significant difference in DIMBOA content could be detected only between P38A79 and PR36V52 hybrids, with higher DIMBOA content in the latter. There were no significant differences among the other studied hybrids. The DIBOA content of hybrids did not differ significantly.

\section{Temporal changes of cyclic hydroxamic acids: organ age and plant age}

The hydroxamate content of the first leaf was measured at each sampling time, allowing us to examine the effect of organ age on hydroxamate content (Figures S1 and S2*). The initial DIMBOA content decreased significantly $(\mathrm{p}<0.01)$ by the end of the investigation period ( $21^{\text {st }}$ day) (Figure S1). The effect of the hybrid was also significant $(p=0.026)$. The change in the DIMBOA content in the P9494 hybrid was different, being initially lower than those of the others.

*Further details about the Electronic Supplementary Material (ESM) can be found at the end of the article. 
In the first leaves, the temporal changes of the DIBOA content were significant $(\mathrm{p}<0.01)$ but less uniform, and showed different trends compared to those for DIMBOA content (Figure S2). The effect of the hybrid was also significant $(\mathrm{p}<0.01)$. The DIBOA content of PR38A79 hybrid decreased with ageing, similar to changes in DIMBOA content. The DIBOA content of the first leaves of the P9494, PR36K67 and PR36V52 hybrids decreased by the $15^{\text {th }}$ day, and then increased towards the end of the sampling period ( $21^{\text {st }}$ day). The DIBOA content of the P9578 hybrid changed irregularly during the study.

The effect of plant age on hydroxamate content was also investigated. The DIMBOA and DIBOA contents in the youngest parts were examined in each hybrid according to plant ages (Figures S3 and S4). DIMBOA content of the youngest leaves showed a significant $(\mathrm{p}<0.01)$ drop by the $9^{\text {th }}$ day for all examined hybrids (Figure S3). Among the studied hybrids, significant differences were found $(\mathrm{p}<0.01)$ considering their DIMBOA contents, depending on time. The amount of DIMBOA decreased quickly in the PR38A79 and P9578 hybrids and became lower than in the other hybrids.

The DIBOA content $(\mathrm{p}<0.01)$ was also significantly affected by plant age and hybrid. Except for the P9578 hybrid, the highest DIBOA content was measured at the youngest age and dropped down to a minimal concentration by the $9^{\text {th }}$ day. The DIBOA content of the young leaves increased significantly between the ages of 15 to 21 days.

The DIMBOA content of young root parts also significantly decreased over time $(\mathrm{p}<0.01)$ and among the studied hybrids significant differences were recorded $(\mathrm{p}<0.01)$. The DIMBOA content of the PR36K67 hybrid was higher over the entire investigated period. The initial decrease of the hydroxamate content in the P9578 and P9494 hybrids was not detectable (Figure S5).

An opposite and significant $(\mathrm{p}<0.01)$ trend was recognized in the case of DIBOA content. The amount of DIBOA in the PR38A79 and P9578 hybrids initially remained constant and then increased significantly towards the end of the study period $(p<0.01)$. The other hybrids did not show a consistent temporal change. The DIBOA content of the P9494 hybrid was significantly higher, based on the average of four measurements $(\mathrm{p}<0.01)$ (Figure S6).

\section{Discussion}

\section{Cyclic hydroxamic acid content of hybrids}

The hydroxamate content of both shoots and roots were significantly different in the cases of all five hybrids and showed different patterns in time for the different organs. In the shoots, the P9494 hybrid represented the highest value, while in the case of roots, the PR36K67 hybrid showed the highest DIMBOA content. Regarding DIBOA content, this was the highest in both the shoots and roots of the P9494 hybrid.

The DIMBOA contents of both shoots and roots were, in order of magnitude, higher than the DIBOA contents of all the studied hybrids. Regarding resistance capacity, there is no data on the effectiveness of these compounds, so we assumed that they were effec- 
tive according to their amounts; thus, DIMBOA is much more important than DIBOA in the self-defense of maize.

There was no obvious correlation between the hydroxamate contents of the shoots and the roots. At least two different explanations might clarify these results. First, considering the age of the sampled organs, there were differences between the shoot and root samples. In our case, shoot samples were taken from leaves of different ages, while root samples consisted of only the youngest root parts. It is well known that both the age of the organ and the plant affect the hydroxamate content (Toldiné Tóth 1984; Copaja et al. 1991; Reberg-Horton et al. 2005; Elek et al. 2013). An alternative explanation could be that the hydroxamate contents of maize shoots and roots does not correlate. Wu et al. (2001) and Copaja et al. (2006) detected the same phenomenon in rye and wheat, respectively.

The hydroxamate content of the youngest leaves, which were sampled at their first appearance, did not show significant differences, except for the DIMBOA contents of the P38A79 and PR36V52 hybrids, with higher values in the latter. The differences in the hydroxamate content of all measured leaves and the youngest ones varied among the five investigated hybrids. This indicates that the effect of the age of the organ manifests differently in various hybrids. The difference in hydroxamate content in all measured samples was a result of the different initial hydroxamate contents and different trends of its temporal changes.

The hydroxamate content of these five hybrids was studied earlier in a field experiment in 2012. That study also found significant differences among hybrids (Makleit et al. 2012; Nagy et al. 2013). The total hydroxamate content was determined and significant differences were recorded only in cases of the younger shoots ( $<12$ days). The total hydroxamate contents of P9578, PR36K67 and PR36V52 hybrids were higher, while the P9494 hybrid had medium and the PR38A79 hybrid had the lowest hydroxamate contents. The results of the field and the laboratory studies cannot be compared because of the differences in environmental conditions. The differences in the hydroxamate contents of hybrids could be established in both field and laboratory conditions, and may provide a basis for the selection of the hybrid with the highest resistance.

\section{The effect of organ age on hydroxamate content}

Investigation of the relation between the resistance against pests or pathogens, the hydroxamate contents of different maize hybrids and the effects of different parameters on it are thoroughly studied topics in plant pathophysiology. Since published research has generally focused on the changes in the hydroxamate content of a given organ (root or leaves) or the whole shoot of a given hybrid, there is not enough data that can be used directly in the selection of most resistant hybrids.

The DIMBOA content of the first leaf decreased significantly by the end of the investigation period. The characteristics and the value of the decrease were almost similar in all hybrids. For the first node, Toldiné Tóth (1984) reported on the decrease of hydroxamate content by age of the organs, while in case of different leaves, Morse et al. (1991) showed the same results. Thus, the observed trends in DIMBOA content can be consid- 
ered to be a general phenomenon in maize. The temporal changes of the DIBOA content, which were also significant, but less uniform, showed different trends. One of the hybrids (PR38A79) showed similar change to the DIMBOA content, but the DIBOA content of the other hybrids changed in different ways, mostly increasing before the end of the study period. Cambier et al. (2000) also observed that, contrary to other hydroxamates, the HDMBOA-Glc (N-O-methylated DIMBOA-Glc) content in maize roots remained stable between the ages of 6-20 days. Thus the decreasing of hydroxamate content seems not to be a generalized phenomenon for all hydroxamates.

The role of hydroxamates in maize resistance depends rather on the DIMBOA, than on the DIBOA content, and its efficiency especially depends on the rate of decrease in the plant organs. In this study, we could not demonstrate differences in temporal changes of DIMBOA content among the hybrids. In the cases of other crop species (e.g. wheat and rye), the separation of various leaves is rather difficult, so in these cases, only the effect of plant age on the hydroxamate content can be investigated (Copaja et al. 1991; RebergHorton et al. 2005).

\section{The effect of plant age on hydroxamate content}

Investigation of the quantity of hydroxamates and its changes in the youngest plant organs at different stages of development is relevant because pathogens and pests attack mainly the youngest parts. Therefore, the DIMBOA and DIBOA contents in the youngest parts were examined in each hybrid, according to plant age. Among the studied hybrids, significant differences were found for both studied compounds.

A significant drop in the DIMBOA contents of the youngest leaves was established by the $9^{\text {th }}$ day of the experiment for all hybrids. This drop was higher in the PR38A79 and P9578 hybrids, leading to lower final hydroxamate contents. Cambier et al. (2000) also demonstrated such a decreasing trend of hydroxamate content, both in shoots and even in maize roots. The role of DIMBOA in plant defense becomes less significant with plant development, so this also strengthens its role in young plant defense mechanisms, especially at early stages. In this case, other secondary metabolites - cell wall components, flavonoids, protease inhibitors and proteinases - must take over the function of hydroxamates (Meihls et al. 2012). Moreover, the resistance of maize lines and hybrids to the European corn borer (Ostrinia nubilalis Hübner 1796) did not show a correlation to hydroxamate content (Barry et al. 1994); therefore, study of the relation between resistance and hydroxamate content in cases of especially older plants may lead to erroneous conclusions.

In the cases of four hybrids, the highest DIBOA content was measured at the youngest age and dropped down to a minimal value by the $9^{\text {th }}$ day. Afterwards, the DIBOA content increased significantly between the ages of 15 to 21 days. The DIBOA content of the P9578 hybrid fluctuated during the sampling period. Such kinds of unusual trends in hydroxamate content were also detected in the case of HDMBOA-Glc (Cambier et al. 2000).

In the case of the roots, the place of sampling, namely which root part is the object of the study, dramatically determines the measured hydroxamate content (Xie et al. 1991a). 
The growing of a plant in a nutrient solution facilitated exact and correct sampling from the youngest parts.

The DIMBOA and DIBOA contents of young root parts significantly changed over time, following opposite trends. DIMBOA content decreased, while the amount of DIBOA increased or changed irregularly. The temporal changes of hydroxamate content of organ differences of the same five hybrids were found in a field study, as also reported by Makleit et al. (2012) and Nagy et al. (2013). In the case of field experiments, the temporal changes of total hydroxamate content were determined with the average of studied hybrids and significant decrease was measured both in shoots and roots. Understanding temporal changes of different hydroxamic acids, the former result became more comparable and provided a useful tool for the development of experimental methodology (e.g., the selection of the appropriate sampling time).

The reduced hydroxamate synthesis of the older organs and even the young organs of older plants can be explained by the trade-off between growth and defense. In the current study, the plants were free from any stressors, so the production of hydroxamates could be decreased. In contrary a pest attack can induce both the rise of hydroxamate synthesis, even in the older organs in later growth stages (Gutierrez et al. 1988), and the translocation of hydroxamates from other plant parts (Gianoli, Niemeyer 1997).

It may be concluded that the above presented comparative study may establish a protocol for examining candidate varieties from the point of view of plant self-defense. The observed differences of hydroxamate content (as a function of e.g., variety, organ development, time) opens the door to determining appropriate methods of selection of suitable hybrids and new breeding lines with higher hydroxamate contents for better performance in pest resistance, where relevant.

\section{Acknowledgements}

László Somsák and Attila Kiss (University of Debrecen, Faculty of Science and Technology, Institute of Chemistry, Department of Organic Chemistry) are acknowledged for their valuable advices and help during the preparative work. Thanks are due to Dieter Sicker (University of Leipzig, Institute for Organic Chemistry, Germany) for the additional DIMBOA sample for comparison of our own standards. The authors wish to thank the financial support of Pioneer Hi-Bred ZRt (Hungary).

\section{References}

Abel, C.A., Berhow, M.A., Wilson, R.L., Binder, B.F., Hibbard, B.E. 2000. Evaluation of conventional resistance to European corn borer (Lepidoptera: Crambidae) and western corn rootworm (Coleoptera: Chrysomelidae) in experimental maize lines developed from a backcross breeding program. J. Econ. Entomol. 93:1814-1821.

Assabgui, R.A., Arnason, J.T., Hamilton, R.I. 1993. Hydroxamic acid content in maize (Zea mays) roots of 18 Ontario recommended hybrids and prediction of antibiosis to the western cornrootworm, Diabrotica virgifera virgifera LeConte (Coleoptera, Chrysomelidae). Can. J. Plant Sci. 73:359-363. 
Assabgui, R.A., Hamilton, R.I., Arnason, J.T. 1995a. Hydroxamic acid content and plant development of maize (Zea mays $\mathrm{L}$ ) in relation to damage by the western corn rootworm, Diabrotica virgifera virgifera LeConte Can. J. Plant Sci. 75:851-856.

Assabgui, R.A., Arnason, J.T., Hamilton, R.I. 1995b. Field evaluations of hydroxamic acids as antibiosis factors in elite maize inbreds to the Western corn rootworm (Coleoptera: Chrysomelidae). J. Econ. Entomol. 88:1482-1493.

Barry, D., Alfaro, D., Darrah, L.L. 1994. Relation of European corn borer leaf-feeding resistance and DIMBOA content in maize. Environ. Entomol. 23:177-182.

Cambier, V., Hance T., Hoffmann, E. 2000. Variation of DIMBOA and related compounds content in relation to the age and plant organ in maize. Phytochem. 53:223-229.

Copaja, S.V., Niemeyer, H.M., Wratten, S.D. 1991. Hydroxamic acid levels in Chilean and British wheat seedlings. Ann. Appl. Biol. 118:223-227.

Copaja, S.V., Villarroel, E., Bravo, H.R., Pizarro, L., Argandona V.H. 2006. Hydroxamic acids in Secale cereale L. and the relationship with their antifeedant and allelopathic properties. Z. Naturforsch. C 61:670-676.

Elek, H., Smart, L., Martin, J., Ahmad, S., Gordon-Weeks, R., Anda, A., Welham, S., Werner, P., Pickett, J. 2013. Hydroxamic acids in Aegilops species and effect on Rophalosiphum padi behavior and fecundity. Bull.Insectol. 66(2):213-220.

Gianoli, E., Niemeyer, H.M. 1997. Lack of costs of herbivory-induced defenses in a wild wheat: integration of physiological and ecological approaches. Oikos 80:269-275.

Gutierrez, C., Castanera, P., Torres, V. 1988. Wound-induced changes in DIMBOA (2,4-dihydroxy-7-methoxy-2H-1,4-benzoxazin-3(4H)-one) concentration in maize plants caused by Sesamia nonagrioides (Lepidoptera: Noctuidae). Ann. Appl. Biol. 113(3):447-454.

Hartenstein, H., Lipmann, T., Sicker, D. 1992. An efficient procedure for the isolation of pure 2,4-dihydroxy7-methoxy-2H-1,4-benzoxazin-3(4H)-one (DIMBOA) from maize. Indian J. Heterocycl. Chem. 2:75-76.

Ketskeméty, L., Izsó, L., Könyves-Tóth, E. 2011. Introduction to the IBM SPSS Statistics software system. Artéria Stúdió Kft; Budapest, Hungary: 576 pp. (In Hungarian)

Long, B.J., Dunn, G.M., Routley, G. 1974. Rapid procedure for estimating cyclic hydroxamate (DIMBOA) concentration in maize. Crop Sci. 14:601-603.

Lyons, P.C., Hipskind, J.D., Wood, K.V., Nickolson, R.L. 1988. Separation and quantification of cyclic hydroxamic acids and related compounds by high-pressure liquid chromatography. J. Agric. Food Chem. 36:57-60.

Makleit P., Nagy, A., Székács, A., Fónagy, A. 2012. Comparison of Pioneer maize hybrids’ cyclic hydroxamic acid contents. (In Hungarian with English abstract) Növénytermelés 61(4):107-118.

McMullen, M.D., Frey, M., Degenh, J. 2009. Genetics and Biochemistry of Insect Resistance in Maize. In: Bennetzen J.L., Hake, S.C. (eds). Handbook of Maize: Its Biology. Springer Science Business Media, Germany. pp. 271-289.

Meihls L.N., Kaur, H., Jander, G. 2012. Natural variation in maize defense against insect herbivores. Cold Spring Harbor Symposia on Quantitative Biology 77, 269-283. Originally published online December 6, 2012. Access the recent version: doi:10.1101/sqb.2012.77.014662.

Morse, S., Wratten, S.D., Edwards, P.J., Niemeyer, H.M. 1991. Changes in the hydroxamic acid content of maize leaves with time and after artificial damage; implications for insect attack. Ann. Appl. Biol. 119:239249.

Nagy, L., Nagy, L.G., Makleit, P. 2013. Cyclic hydroxamic acid content of maize hybrids measured by HPLCmethod. Poster presented at: 48th Croatian and 8th International Symposium of Agriculture. Osijek, Croatia. 2013.02.17-22.

Niemeyer, H.M. 2009. Hydroxamic acids derived from 2-Hydroxy-2H-1,4-Benzoxazin-3(4H)-one: key defense chemicals of cereals. J. Agric. Food Chem. 57(5):1677-1696.

Reberg-Horton, S.C., Burton, S.C., Danehower, D.A., Ma, G.Y., Monks, D.W., Murphy, J.P., Ranells, N.N., Williamson, J.D., Creamer, N.G. 2005. Changes over time in the allelochemical content of ten cultivars of rye (Secale cereale L.). J. Chem. Ecol. 31:179-193.

Reiczigel, J., Harnos, A., Solymosi, N. 2007. Biostatistics for not professional statisticians. Pars Kft. Nagykovácsi, Hungary: 1-455. pp. (In Hungarian) 
Reid, L.M., Arnason, J.T., Nozzolillo, C., Hamilton, R.I. 1991. Laboratory and field-resistance to the European corn borer in maize germplasm. Crop Sci. 31:1496-1502.

Sytykiewicz, H. 2014. Differential expression of superoxide dismutase genes in aphid-stressed maize (Zea mays L.) seedlings. PLoS One 9(4): e94847. DOI:10.1371/journal.pone.0094847.

Sytykiewicz, H., Chrzanowski, G., Czerniewicz, P., Sprawka, I., Łukasik, I., Goławska, S., Sempruch, C. 2014. Expression profiling of glutathione transferase genes in Zea mays (L.) seedlings infested by cereal aphids. PLoS One 9(11): e111863. DOI:10.1371/journal.pone.0111863.

Sytykiewicz, H. 2016. Deciphering the role of NADPH oxidase in complex interactions between maize (Zea mays L.) genotypes and cereal aphids. Biochem. Biophys. Res. Commun., 476: 90-95. DOI: 10.1016/j. bbrc.2016.05.050.

Toldiné Tóth, É. 1984. Relationship between DIMBOA content and Helmisthosporium turcicum resistance in maize. Növénytermelés 33:213-218.

Treeby, M., Marschner, H., Römheld, V. 1989. Mobilisation of iron and other micronutrient cations from a calcareous soil by plant borne, microbial and synthetic chelators. Plant and Soil 114:217-226.

Wu, H.W., Haig, T., Pratley, J., Lemerle, D., An, M. 2001. Allelochemicals in wheat (Triticum aestivum L.): production and exudation of 2,4-dihydroxy-7-methoxy-1,4-benzoxazin-3-one. J. Chem. Ecol. 27:16911700.

Xie, Y.S., Arnason, J.T., Philogéne, B.J.R., Atkinson, J., Morand, P. 1991a Distribution and variation of hydroxamic acids and related compounds in maize (Zea mays) root system. Can. J. Bot. 69(3):677-681.

Xie, Y.S., Atkinson, J., Arnason, J.T., Morand, P., Philogene, B.J.R. 1991b. Separation and quantification of 1,4-benzoxazin-3-ones and benzoxazolin-2-ones in maize root extract by highperformance liquid chromatography. J. Chromatogr. 543:389-395.

Xie, Y.S., Arnason, J.T., Philogene, B.J.R., Olechowski H.T., Hamilton, R.I. 1992. Variation of hydroxamic acid content in maize roots in relation to geographic origin of maize germplasm and resistance to western corn rootworm (Coleoptera: Chrysomelidae). J. Econ. Entomol. 85:2478-2485.

Zuniga, G.E., Massardo, F. 1991. Hydroxamic acid content in undifferentiated and differentiated tissues of wheat. Phytochem. 30:3281-3283.

\section{Electronic Supplementary Material (ESM)}

Electronic Supplementary Material (ESM) associated with this article can be found at the website of CRC at https://akademiai.com/loi/0806

Electronic Supplementary Figure S1. Changes of the mean DIMBOA content of the 1st leaf of the studied hybrids during the experiment. H1 = PR38A79; H2 = P9578; H3 = P9494; H4 = PR36K67; H5 = PR36V52

Electronic Supplementary Figure S2. Changes of the mean DIBOA content of the $1^{\text {st }}$ leaf of the studied hybrids during the experiment. $\mathrm{H} 1=$ PR38A79; H2 = P9578; H3 = P9494; H4 = PR36K67; H5 = PR36V52

Electronic Supplementary Figure S3. Changes of the mean DIMBOA content of the youngest leaves sampled at their first appearance during the study of the five hybrids: H1 = PR38A79; H2 = P9578; H3 = P9494; H4 = PR36K67; H5 = PR36V52

Electronic Supplementary Figure S4. Changes of the mean DIBOA content of the youngest leaves sampled at their first appearance during the study of the five hybrids: H1 = PR38A79; H2 = P9578; H3 = P9494; H4 = PR36K67; H5 = PR36V52

Electronic Supplementary Figure S5. Changes of the mean DIMBOA content of the youngest part of the root during the study of the five hybrids. H1 = PR38A79; H2 = P9578; H3 = P9494; H4 = PR36K67; H5 = PR36V52

Electronic Supplementary Figure S6. Changes of the mean DIBOA content of the youngest part of the root during the study of the five hybrids. H1 = PR38A79; H2 = P9578; H3 = P9494; H4 = PR36K67; H5 = PR36V52 